\title{
Trayectoria escolar de una alumna con sordera en una primaria rural mexicana: generando posibilidades de aprendizaje y desarrollo
}

\author{
Gabriela Begonia Naranjo Flores*
}

\begin{abstract}
Resumen
Se presenta un estudio etnográfico que aborda las condiciones de escolarización de una alumna con sordera en una escuela primaria rural mexicana ${ }^{1}$. Entrevistas con su maestra y la Directora, permiten reconstruirla trayectoria escolar de la alumna, mientras que la observación y videograbación de clases de ciencias naturales, brindó un acercamiento alas interacciones sociales y académicas que establece. Desde un andamiaje teórico que recupera la perspectiva histórico-cultural de Vigotsky, el análisis de datos sobre esa trayectoria muestra la producción de diferentes condiciones para la atención educativa de la alumna y sus efectos en sus posibilidades de desarrollo y aprendizaje.
\end{abstract}

Palabras clave: Sordera; Inclusión; Escuela Rural; Ciencias Naturales; Vigotsky.

\section{School trajectory of a student with deafness in a rural Mexican primary school: generating possibilities for learning and development}

\begin{abstract}
This ethnographic study addresses the schooling conditions of a student with deafness in a rural Mexican primary school. Interviews with their teacher and school director, allow reconstructing the student's school trajectory, while observation and video recording of natural science lessons, allow document the social and academic interactions she establishes. The theoretical scaffolding recovers the Vigotsky's historical-cultural perspective, so the analysis of the data shows the production of different conditions for the educational attention of the deaf student and its effects on her possibilities of development and learning.

Keywords: Deafness; Inclusion; Rural School; Natural Sciences; Vigotsky.
\end{abstract}

\section{Introducción}

En el marco de la reforma global de su sistema educativo en los noventa, México emitió, a través de la Ley General de Educación, un artículo específicamente dedicado a la educación especial. El artículo 41 asignó a esta modalidad educativa, entre otras cosas, la responsabilidad de "propiciar" la integración de los menores con discapacidad a los planteles de educación básica regular (MÉXICO, 1993, p. 48). Después de veinticinco años de haberse emitido, este artículo ha sufrido una serie de reformas, pero mantiene el mandato de "favorecer" su escolarización en escuelas "comunes", tal como se muestra en el siguiente fragmento de su última reforma de enero de 2016:

Tratándose de personas con discapacidad, con dificultades severas de aprendizaje, de conducta o de comunicación, se favorecerá su atención en los planteles de educación básica, sin que esto cancele su posibilidad de acceder a las diversas modalidades de educación especial atendiendo a sus necesidades. Se realizarán ajustes razonables y se aplicarán métodos, técnicas, materiales específicos y las medidas de apoyo necesarias para garantizar la satisfacción de las necesidades básicas de aprendizaje de los alumnos y el máximo desarrollo de su potencial para la autónoma integración a la vida social y productiva (MÉXICO, 2016, p. 2).

Como efecto de la política de "integración" y más tarde de "inclusión" educativa, se ha incrementado la presencia de alumnos con discapacidad en aulas comunes, por lo que resulta fundamental hacer un seguimiento de qué es lo que está ocurriendo con ellos en estos escenarios. En otras palabras, es imperativo documentar qué tanto y cómo se está haciendo efectivo su derecho a la educación, lo cual no sólo significa acceder y permaneceren la escuela de su comunidad, sino sobre todo satisfacer sus necesidades básicas de aprendizaje (UNESCO, 1990), para estimular el desarrollo pleno de sus potencialidades. En México existe escasa investigación relacionada con este tema y la que hay brinda panoramas generales que alertan sobre las serias problemáticas asociadas a la escolarización de estos alumnos. Por ejemplo, Juárez, Comboni y Garnique (2010, p.66), analizan

*Correo Electrónico: gnaranjo@upn.mx 
cómo se han concretado las políticas para la educación especial desde que la integración fue formulada y señalan lo siguiente:

[...] no capacitar a los maestros y maestras para la atención de niños con NEE (necesidades educativas especiales) en las "aulas regulares" hizo que estos últimos fueran prácticamente marginados de las actividades cotidianas de los otros alumnos, y considerados como un obstáculo para el aprendizaje de los demás, así como para el buen desempeño de los docentes, con el consiguiente descuido de su aprendizaje por falta de atención o de adaptaciones curriculares adecuadas a sus necesidades especiales de aprendizaje. Esta situación dio como resultado un aprendizaje insuficiente $y$, sobre todo, un fracaso en el proceso de integración.

En otro trabajo, Meza (2015)aborda la situación actual de la educación de niños y niñas con discapacidad en México, presentando un apartado dedicado a un breve pero significativo análisis de cómo se ha implementado la educación inclusiva en este país. A partir de su análisis, cierra su texto con un fuerte señalamiento:

El proyecto de inclusión educativa en México sigue la tendencia observada por Dussel (2000), Littlewood (2005) y Popkewitz y Lindblad (2005), de centrarse en acciones de acceso a las escuelas para determinados grupos de excluidos, sin prestar atención a los mecanismos mediante los cuales, con base en un discurso de inclusión, se están generando nuevas formas de excluir a ciertos sectores de la sociedad del ejercicio de sus derechos como ciudadanos. (MEZA, 2015, p. 81)

Trabajos como los citados son sumamente valiosos, pues muestran de manera general procesos asociados a la escolarización de niños con discapacidad. Sin embargo, también son relevantes los estudios que se adentran a la cotidianidad de la escuela, sobre todo para dar cuenta de la complejidad implicada en su atención educativa en entornos comunes. Los estudios de este tipo también son escasos, pero los que hay, resaltan la importancia de las condiciones materiales y humanas en ese proceso, así como el papel de las formas de participación de los agentes involucrados. Por ejemplo, a partir de un estudio etnográfico previo en un aula de escuela primaria en México (NARANJO, 2017), documento cómo un docente busca favorecer el aprendizaje y la participación de su alumno ciego durante sus clases, mientras que doy cuenta tanto de las condiciones que favorecen como las que dificultan este trabajo, concluyendo, en otras cosas, que las políticas de educación inclusiva no generan las condiciones necesarias y suficientes para viabilizar su implementación en las escuelas.

En un estudio de Brasil, Soares y Lacerda (2007) analizan la construcción de la identidad de un alumno sordo mediante su inserción en el aula regular, con compañeros oyentes. Entre otras cosas, las autoras señalan que el modo en que la escuela está organizada pedagógicamente, no toma en cuenta lo que implica la sordera en su complejidad, por lo que no atiende las necesidades específicas del alumno. Así, encuentran que la constitución de la identidad de este niño como sordo se ve afectada al interactuar sólo con sus pares oyentes, pues llega incluso a actuar como si él también lo fuera. A partir de esto, cuestionan el que se considere que un alumno está incluido sólo porque es hábil socializando con los oyentes y convocan a reflexionar sobre la importancia de su interacción temprana tanto con sordos como con oyentes.

Datos como los que aportan los estudios referidos, enfatizan la importancia de indagar de manera más sistemática las formas específicas en que se están atendiendo las necesidades educativas de los alumnos con discapacidad en la cotidianidad de las escuelas. Es ahí donde las propuestas de educación inclusiva se viabilizan o no; donde podemos desmontar discursos que sólo apelan al compromiso de los participantes, sin comprometer acciones de política que realmente generen condiciones para hacerlas efectivas; y donde podemos visualizar caminos posibles para lograrlo. En este sentido, el propósito en este artículo es analizar la trayectoria escolar de una alumna con sordera profunda en una escuela primaria rural mexicana. El concepto de trayectoria es útil, en la medida que:

Permite enlazar la biografía con las condiciones institucionales $\mathrm{y}$, en lo que atañe al campo educativo, permite analizar de qué manera las condiciones institucionales del sistema escolar intervienen en la producción de las trayectorias escolares, asunto que suele quedar oculto bajo la importancia que se asigna a las difíciles condiciones en que se desarrollan las vidas de numerosos niños, niñas, adolescentes y jóvenes [...] (TERIGI, 2014, p. 75)

La reconstrucción y análisis de la trayectoria escolar de una alumna con sordera permitirá en este trabajo analizar diferentes condiciones generadas por el sistema escolar y los actores locales en una escuela 
primaria de Oaxaca y algunos de sus impactos en el desarrollo y aprendizaje de dicha alumna.

\section{Referentes teóricos y conceptuales}

La teoría sociocultural de Vygotsky brinda el andamiaje teórico pertinente para el propósito de este estudio, ya que pone de relieve la importancia de las condiciones materiales, sociales y culturales de vida de los individuos en el desarrollo pleno de sus potencialidades:

La principal tesis de Vygotsky suena paradójica: para explicar las formas más complicadas de la vida consciente del hombre es imprescindible salir de los límites del organismo, buscar los orígenes de esta vida consciente y del comportamiento "categorial", no en las profundidades del cerebro ni en las profundidades del alma, sino en las condiciones externas de la vida y, en primer lugar, de la vida social, en las formas histórico sociales de la existencia del hombre. (LURIA, 1984, p. 22)

Sin duda una contribución fundamental de Vygotsky (1979), fue destacar la naturaleza sociocultural de todo tipo de actividad humana, incluyendo al aprendizaje. La actividad de un individuo, por más aislada o "interna" que parezca, está configurada por la historia de las relaciones sociales en las que participa y no puede ser entendida al margen de las mismas. Este planteamiento, así como su ulterior desarrollo, cuestiona la dicotomía que suele establecerse entre la actividad individual y la social, así como entre la actividad interna (mental o psicológica) y la externa, planteando más bien la indisoluble y compleja relación esos diferentes aspectos de la actividad humana.

Pero el genio de Vigotsky (1979), además reveló el "mecanismo" básico que posibilita ese nexo indisoluble entre los diferentes tipos de actividad humana: su mediación a través de herramientas y sistemas de signos. Propuso que los signos del lenguaje median la acción, primero de forma ínterpsicológica y luego, de forma intra-psicológica. Con esta idea, Vigotsky, hizo dos aportaciones sumamente importantes. La primera, resaltar el papel de los procesos inter-personales en la formación y desarrollo de los procesos mentales, eliminando así la oposición que frecuentemente ha llevado a fragmentar el estudio de la vida social:

[...] hay una relación inherente entre la actividad externa e interna, pero en forma de una relación genética en la que el punto principal es cómo son creados los procesos psicológicos internos como resultado de la exposición del niño a lo que Vygotsky denominaba "formas culturales maduras de comportamiento". (VIGOTSKY, 1981, citado por WERTSCH, 1988, p.80)

Desde la perspectiva sociocultural, la actividad mental es producto de las relaciones sociales y está conformada histórica y culturalmente. A su vez, ésta actividad define, regula y transforma las acciones de los individuos desplegadas hacia su entorno.

La segunda aportación de Vigotsky fue haber llamado la atención hacia el papel primordial de los instrumentos que median la acción, tales como las herramientas y los signos del lenguaje. De hecho, la conceptualización sobre estos instrumentos constituye la base sobre la cual fundamenta la unidad de la actividad interna y externa. Vigotsky, retoma la tesis de Engels respecto a que el uso de herramientas durante el trabajo humano cambia la naturaleza y a la vez produce transformaciones sobre sí mismo: "La especialización de la mano significa la herramienta y ésta presupone la actividad específicamente humana, la reacción transformadora del hombre sobre la naturaleza... mediante sus cambios, la hace servir a sus fines, la domina" (ENGELS, 1940, citado por COLE; SCRIBNER, 1979, p. 26) De esta tesis se desprende que una herramienta es un objeto del entorno modificado y utilizado por un individuo para el logro de sus fines.

Vigotsky extendió la categoría de herramienta para incluir el uso de sistemas de signos elaborados cultural e históricamente, tales como el lenguaje, los números y la escritura. Además afirmó que la internalización de estos sistemas produce transformaciones tanto en la actividad mental como en el comportamiento del individuo (COLE; SCRIBNER, 1979). Esto es, Vigotsky incluyó a los signos y a las herramientas materiales o técnicas en la misma categoría de mediación, pero también estableció claramente sus diferencias. Mientras que una herramienta se orienta a la transformación del objeto externo de su actividad, un signo:

[...] no cambia nada en el objeto de una operación psicológica. Un signo es un instrumento para influir psicológicamente en la conducta, tanto si se trata de la conducta del otro como de la propia; es un medio de actividad interna dirigida al dominio de los propios humanos. Un signo está interiormente dirigido (VIGOTSKY, 1960, citado por WERTSCH, 1988, p. 94) 
Vigotsky desarrolló una concepción sobre las herramientas psicológicas, semióticamente orientada, es decir, destacando su "sentido de poseedor de significado" (VIGOTSKY, 1979, citado por WERTSCH, 1988, p. 34) y puso como ejemplo de ellas y de sus sistemas complejos al lenguaje, los sistemas para contar, las técnicas mnemónicas, los sistemas de símbolos algebraicos, esquemas, escritos, diagramas, mapas y todo tipo de signos convencionales (WERTSCH, 1988).

Desde los planteamientos de Vigotsky queda claro que toda acción humana es siempre mediada, se realiza a través de los sistemas de signos y herramientas que los escenarios culturales, históricos e institucionales hacen disponibles a quienes participan en ellos y, por tanto, está sujeta en grados $\mathrm{y}$ formas variables a las determinaciones que estos escenarios imponen.

Lo que permite que los individuos se apropien de determinados sistemas de signos y herramientas es su participación en los escenarios culturales, históricos e institucionales donde dichos sistemas se utilizan. De esta manera, el análisis en este trabajo, está orientado a examinar cómo las condiciones de escolarización posibilitan o constriñen la participación de una alumna con sordera en la vida social y académica de su escuela y si esa forma de participación le permite apropiarse de los sistemas de signos y herramientas necesarios para su aprendizaje y desarrollo.

\section{Metodología}

La etnografía es el enfoque teórico metodológico que de manera general orientó la realización de este estudio, ya que permite documentar procesos no documentados de la vida cotidiana de las personas y grupos sociales ahí donde estos ocurren, tratando de comprenderlos desde sus propias perspectivas, sin categorizaciones previas, pues estas se construyen a partir de los datos empíricos en diálogo con la teoría (ROCKWELL, 1986, 2009).Desde la etnografía, las aulas pueden ser estudiadas como un medio social y culturalmente organizado para el aprendizaje, reconstruyendo los fenómenos de la interacción cotidiana y sus significados desde la perspectiva de los actores educativos. Este enfoque es congruente con la perspectiva metodológica de Vigotsky, quien planteó que los contextos adecuados para estudiar la vida consciente del ser humano, son los ambientes reales en los que ésta se desenvuelve, si es que se quiere vislumbrar posibilidades de transformación y mejora de la vida humana (RIVIÉRE, 1985).

Los datos que se consideran para el análisis fueron recabados en el contexto de un estudio más amplio, cuyo propósito general es analizar las prácticas de enseñanza y aprendizaje de ciencias naturales en aulas de escuelas primarias rurales de diferentes entidades de la República Mexicana. El caso que se presenta, fue documentado en una escuela rural de Oaxaca, localizada en una comunidad de poco más de 1900 habitantes. Las familias, de nivel socioeconómico bajo, se dedican básicamente al trabajo asalariado en una zona turística cercana a la comunidad, a la pesca y al comercio informal.

El caso que se presenta es el de Itzel $^{2}$, una alumna de sexto grado de primaria, con 12 años de edad y sordera profunda, que resulta interesante por las características de su trayectoria escolar. Ella cursó en la misma escuela los seis grados de la primaria y, al momento de la observación, estaba repitiendo el sexto grado. En sus primeros años en esta escuela, Itzel fue escolarizada y atendida, pero sin contar con las condiciones mínimas para garantizar "la satisfacción de sus necesidades básicas de aprendizaje y el máximo desarrollo de su potencial para la autónoma integración a la vida social y productiva", tal como lo plantea el artículo 41 (MÉXICO, 2016, p. 2). En cierto punto de su trayectoria, con la llegada de una nueva Directora escolar, dichas condiciones comenzaron a ser transformadas, generando nuevos escenarios para Itzel. De esta manera, es posible analizar cómo diferentes condiciones de escolarización produjeron impactos diferenciales en las posibilidades de aprendizaje y desarrollo de la niña.

El trabajo de campo incluyó la observación de ocho clases de ciencias naturales, dos por semana, que tuvieron una duración de entre 60 a 120 minutos. El registro de las clases se hizo en diario de campo, videograbaciones y fotografías de diferentes aspectos de la vida del aula. De las ocho sesiones observadas, Itzel estuvo en cuatro, ya que una vez por semana acudía a una institución filantrópica y de asistencia privada para recibir capacitación laboral. También se realizaron entrevistas con la Directora de la escuela y la maestra responsable del grupo, además de algunas conversaciones informales con el Supervisor y una Asesora Técnico Pedagógica, ambos de Educación Especial de esa zona escolar.

\section{Una escolarización con cierto aislamiento}

Desde antes de mi entrada a la escuela, la 
Directora me había comentado que en uno de los grupos a los que entraría a observar había una alumna con sordera profunda. Ya en el campo, los datos sobre la escolarización de Itzel poco a poco comenzaron a fluir y a llamar cada vez más mi atención. Un primer dato que sobresalió es el hecho de que esta niña estuviera repitiendo el sexto grado. La maestra de grupo, que en adelante llamaré Maty, comenta que ella tuvo a cargo el año pasado al grupo de Itzel, de sexto grado, pero que no la aprobaron porque no consideraron que estuviera apta para irse a la secundaria, pues "aún no sabe leer ni escribir. Entonces consideramos que (quedándose otro año) podíamos ayudarle un poquito más." La Directora también aporta datos sobre las razones de esta decisión:

El año pasado Itzel ya estaba en sexto, pero platicamos con la mamá de que ella todavía no había desarrollado las habilidades sociales para, por ejemplo, comunicarse y trasladarse a la secundaria y además de que todavía no había adquirido la lectura y la escritura (DIRECTORA, 23/02/18).

Llamó mucho mi atención el que después de seis años en esa escuela, Itzel aún no supiera leer y escribir, así que las entrevistas con la maestra de grupo y con la Directora, tuvieron como uno de sus propósitos indagar más sobre este asunto. La información que me fueron proporcionando permite reconstruir la trayectoria de la alumna en la escuela.

Itzel aprendió la Lengua de Señas Mexicana (LSM) desde temprana edad en una fundación filantrópica, así que ingreso a la primaria ya con ese manejo. Durante su primer grado, la maestra Maty fue también la responsable de atenderla en su grupo. En ese entonces estuvo recibiendo orientación de una maestra dela fundación ya mencionada sobre cómo podía trabajar con esta alumna. Sin embargo, al parecer, este apoyo no fue muy significativo para la maestra Maty:

Pues yo la tuve en primero. Ella siempre ha ido a una escuela especial en la ciudad, entonces la maestra venía una vez a la semana y ya me compartía cómo podía trabajar con ella. Pero no fue tanto el avance como ahora que están los compañeros de USAER3. (MAESTRA DE GRUPO, 21/02/18)

Cabe señalar que esta maestra nunca recibió alguna formación o capacitación específica para trabajar con alumnos con sordera o alguna otra deficiencia. Y, en general, parece ser que la escuela no sabía cómo hacerlo, aunque si hacían algunos esfuerzos al respecto:

Itzel ya estaba en cuarto. Anteriormente, me comentaron los compañeros de que la maestra que la atendía tenía su cuaderno de lenguaje de señas y sí, si interactuaba un poquito con ella, pero no a las dimensiones de lo que representó o lo que representa el que esté USAER aquí en la escuela. (DIRECTORA, 23/02/18)

Por los comentarios de la maestra Maty y de la Directora, se infiere que la llegada de Unidad de Servicios de Apoyo a la Escuela Regular (USAER) a la escuela marca un antes y un después en la escolarización de Itzel. Hasta ese momento, la niña se mantenía con cierto aislamiento de la comunidad escolar, ya que entablaba pocas interacciones con sus compañeros y maestros: "Con Itzel tenemos la situación de que ella era poco sociable, solamente interactuaba con su mamá, principalmente, y no había una interacción en grupo. Primeramente porque no sabíamos del lenguaje de señas". (DIRECTORA, 23/02/18)

La Directora reconoce que uno de los factores que incidió en ese aislamiento era el desconocimiento de la LSM y la falta de experiencia en el trabajo con alumnos con sordera: "honestamente yo nunca en mi experiencia había tenido una niña con hipoacusia." Por su parte, la maestra Maty reconoce que, como escuela, "no sabemos cómo ayudarla". Y, por la forma en que lo expresa, para ella este es un problema no sólo de los ciclos escolares pasados, sino también del actual. En este sentido, puedo decir que el sistema educativo no había generado las condiciones mínimas necesarias, demandadas en la ley, para la atención de la alumna con discapacidad:

La educación especial deberá incorporar los enfoques de inclusión e igualdad sustantiva. Esta educación abarcará la capacitación y orientación a los padres o tutores; así como también a los maestros y personal de escuelas de educación básica y media superior regulares que atiendan a alumnos con discapacidad, con dificultades severas de aprendizaje, de comportamiento o de comunicación, o bien con aptitudes sobresalientes. (MÉXICO, 2016, p. 2)

Una condición que si fue generada por el sistema educativo mexicano consistió en proporcionar a Itzel un sistema de comunicación y representación alternativo, construido social e 
históricamente por y para las personas con deficiencia auditiva: la LSM. En términos de Vigotsky, esta lengua puede ser considerada como un complejo sistema de signos con el potencial de mediar tanto la actividad mental como social de la niña, favoreciendo el desarrollo, tanto de sus procesos de aprendizaje como de interacción social, los cuales están indisolublemente ligados.

Los sistemas de signos son los medios a través de los cuales es posible la comunicación y, por tanto, la acción concertada entre las personas. A su vez, tal como lo estableció Vigotsky, estos sistemas son producto de las necesidades de la vida social:

[...] En el proceso de vida social, los humanos han creado y desarrollado sistemas extremadamente complejos de vínculos psicológicos sin los cuales la actividad laboral y toda la vida social serían imposibles. Estos instrumentos de conexión psicológica son, por propia naturaleza y por función, signos [...] (VIGOTSKY, 1960, citado por WERTSCH, 1988, p. 107)

Los sistemas simbólicos (verbales y no verbales), al estar constituidos por signos con significados más o menos compartidos en una cierta comunidad, permiten la comunicación entre las personas. En el caso de Itzel, al llegar a la primaria ya contaba con un sistema de signos para comunicarse, una condición fundamental para su desarrollo. Sin embargo, al no ser compartido por los integrantes de la comunidad escolar, dicha comunicación no se produce, permaneciendo la mayor parte del tiempo en un relativo aislamiento. Es como estar en una escuela donde todo mundo habla inglés, siendo únicamente hispanoparlante o hablante de portugués.

La no disposición de un sistema de comunicación compartido con la comunidad escolar incidió también en un limitado aprendizaje de los contenidos curriculares. Particularmente, limitó la apropiación de otras potentes herramientas del pensamiento, tales como la lengua escrita y las matemáticas, necesarias para continuar aprendiendo y participando en muchos aspectos de la vida social y académica. Esto no implica que en los otros espacios institucionales donde estaba siendo atendida Itzel (la asociación filantrópica y de forma más recientepor el equipo de la Supervisión de Educación Especial), no estuviera aprendiendo otras cosas, pues en ellos ha convivido con hablantes en lengua de señas.

\section{Una mirada incluyente}

A partir de la información proporcionada, tanto de la Maestra de Grupo como de la Directora, infiero que las condiciones de escolarización de Itzel han ido cambiando. Ubico como un momento clave para esto, la llegada de la Directora actual a la escuela. Su mirada hacia las diferencias entre los alumnos, su actitud y su compromiso se pusieron en juego para comenzar a generar mejores condiciones para la educación de todos ellos:

Yo con la experiencia como maestra de grupo ya había trabajado con USAER en otra escuela, siempre me enfocaba en esa parte de considerar que todos los alumnos eran diferentes, entonces trato de transmitir esa parte a los maestros, de que, ipues el aula es diversa ¿no?!Que cada niño tiene diferentes estilos, ritmos y canales de aprendizaje. (DIRECTORA, 23/02/18)

En consonancia con lo anterior, el proyecto escolar ${ }^{4}$, elaborado por el colectivo docente bajo el liderazgo de la Directora, contempla acciones para favorecer una perspectiva respetuosa e incluyente de la diversidad. Por ejemplo, con los alumnos implementan estrategias para favorecer el aprendizaje colaborativo, el trabajo en equipos, el que compartan y que haya respeto entre ellos, entre otras cosas. Como política en la escuela, todos los alumnos, independientemente de sus características o situaciones, son incluidos en las actividades cívicas y sociales, pues lo que se pondera son sus posibilidades y no sus imposibilidades:

También hemos tratado de incluir a Itzel, por ejemplo, cuando a su grupo le toca el homenaje y baila, ¡Itzel baila! Cuando hay algún evento, siempre están presentes (los niños con mayores dificultades) no los marginamos, así de que: ¡no pues tú no puedes! No, al contrario, siempre los hacemos presentes y no solamente a ellos sino que es una dinámica de toda la escuela de que siempre, todos los niños, vayan teniendo sus espacios y sus escenarios. (DIRECTORA, 23/02/18)

La mirada de la Directora, particularmente respecto a Itzel, es también compartida por la maestra Maty, pues al hablar de lo que la niña no ha aprendido o no ha logrado, nunca dio algún indicio de atribuir las dificultades a la alumna. Antes bien, reconoce, como ya se mencionó, que "no sabemos cómo ayudarle". Pero lo que sí sabe, según lo que observé, es cómo hacerla sentir parte del grupo, apreciada y 
respetada. Uno de los días de observación coincidió con que en México se celebrara el Día del amor y la amistad, así que durante el recreo la maestra Maty, en colaboración con la Maestra de Lenguajes, organizaron un pequeño festejo con todo el grupo en el salón. En esa situación fue posible observar a Itzel totalmente incluida y feliz en la interacción con sus compañeros y maestras.

En otra ocasión, Itzel recibió la visita de varios de sus compañeros del grupo anterior, que ya pasaron al siguiente nivel educativo, para festejarla por su cumpleaños. La maestra le permitió salir con ellos a un área afuera del salón, con mesas de madera dispuestas bajo la sombra de varios árboles, para convivir un rato con ellos. Nuevamente, se le observó feliz y animada.

Otro aspecto importante de mencionar sobre el papel de la Directora en la generación de mejores condiciones para la educación de Itzel, está relacionado con sus gestiones para demandar el apoyo de un servicio de educación especial. Cuando la Directora asume la responsabilidad de esta escuela, le llama la atención el que las necesidades educativas de Itzel no se estuvieran atendiendo y que permaneciera en ese nivel de aislamiento. Su experiencia previa como maestra de grupo le permitió actuar en consecuencia: "Cuando yo llego a la escuela no teníamos USAER. Empiezo con la gestión para solicitar el servicio y seis meses después se da que tienen personal y lo envían a esta escuela".

Contar con USAER en la escuela fue un logro importante, sobre todo considerando que no todas las escuelas pueden tenerlo. De hecho, la Directora comenta que varias escuelas de los alrededores no tienen ese servicio, pero como ella fue muy insistente, logró que "le hicieran caso". En medio de la escasez de recursos profesionales de educación especial en la región para dar cobertura a las escuelas, logró que a la suya le dieran prioridad, poniendo en el centro de sus argumentos la necesidad del apoyo por tener a una alumna con sordera profunda. La insistencia fue otro factor que incidió en este logro: "Creo que el Supervisor ya me veía hasta en la sopa", comenta la Directora. Esto fue ratificado por el Supervisor de Educación Especial de esa zona escolar.

La visión de la Directora respecto a las diferencias destaca en el caso analizado como un detonador de pequeñas, pero significativas transformaciones en las condiciones de escolarización de Itzel. De acuerdo con Van Der Veer y Valsiner (2009), Vigotsky consideraba que el potencial de desarrollo de los niños con deficiencias es normal y aspiraba a que éstas fueran vistas como variaciones y no como aberraciones del modelo humano y que pudieran ser miembros integrados y valorados en la sociedad. Esta visión, calificada por los autores citados como utópica, de alguna manera se encuentra en la Directora de la escuela, posiblemente como efecto de su propia historia personal, académica y profesional. Es una visión que en la actualidad cobra vigencia y que resulta imprescindible para transformar los escenarios escolares en espacios socioculturales inclusivos.

La mirada de la Directora y su traducción en acciones concretas, puede verse como una condición favorable para la escolarización de alumnos con discapacidad. Pero no se trata de una voluntad aislada e individual, se trata de una postura construida social, histórica y culturalmente. La entrevista con ella permitió profundizar un poco en su trayectoria profesional, marcada por una militancia muy activa en la lucha magisterial del Estado de Oaxaca y su compromiso por impulsar un proyecto colectivo de transformación de la educación para favorecer a quienes históricamente han sido vulnerados en sus derechos fundamentales. Desde una aproximación sociocultural fundamentada en las ideas de Vigotsky, Wertsch, Tulviste y Hagstrom (1993, p. 343), plantean que la agencia "se extiende más allá de la piel" pues por un lado, puede ser atribuible a grupos más que a individuos y, por otro, es un atributo de individuos operando con recursos mediacionales, social e históricamente constituidos.

\section{Una lengua que comienza a ser compartida}

Tanto para la Directora como para la Maestra de Grupo, la llegada de la USAER a la escuela marcó un antes y un después en las condiciones de escolarización de Itzel. Tener una mirada incluyente no basta, así como tampoco la buena voluntad para incluir a los alumnos con discapacidad. En documentos internacionales que han sido claves en el impulso de las políticas de educación inclusiva, se recomienda la provisión de medidas de apoyo personalizadas y efectivas en entornos que fomenten al máximo el desarrollo académico y social de los alumnos con discapacidad para su plena inclusión (UNESCO, 1994; ONU, 2006). México, que ha suscrito y firmado tales documentos, atendió dicha recomendación desde los noventa, entre otras cosas, con la creación de la USAER. En ese entonces fue definido como un servicio de apoyo a la escuela común para la integración de alumnos con necesidades educativas especiales con 0 
discapacidad y aquellos con capacidades sobresalientes (MÉXICO, 1994). En la actualidad el modelo de atención de este servicio ha sufrido modificaciones, pero su esencia como servicio de apoyo especializado se mantiene.

Desde la perspectiva de la Directora, la USAER ha contribuido con su escuela a cambiar la mirada hacia las diferencias: "la presencia de la USAER y de Itzel en la escuela han hecho que abramos más los ojos a la diversidad. "Pero, de manera muy especial, tanto para la Directora como para la Maestra de Grupo, una acción que ha sido clave para cambiar las condiciones de escolarización de Itzel es la enseñanza dela LSM a varios integrantes de la comunidad escolar. El ciclo escolar anterior al de la observación, la USAER había implementado un taller de LSM con los maestros, lo cual, desde el punto de vista de la Directora, ha sido sumamente importante:

Para comunicarnos con Itzel... nosotros entramos también en esa dinámica de aprender lenguaje de señas...Y ya hemos entrado en que todos los maestros, tratamos de, por ejemplo, en lo más cotidiano, darle el saludo, de ¿cómo estás?, de poder interactuar con ella. (DIRECTORA, 23/02/18)

La Directora se apena y se lamenta de que su propio aprendizaje de LSM esté siendo lento, pues es algo que hay que estar practicando continuamente. En el mismo sentido, la maestra Maty reconoce lo que ha aprendido en ese proceso, pero también las dificultades que enfrenta:

He aprendido algunas señas, pero como no las práctico mucho, ya en el momento que las necesito, pues me acuerdo, o le pregunto a ella (a Itzel) y me dice cómo, o busco mi libro y veo cómo comunicarme con ella. (MAESTRA DE GRUPO, 21/02/18)

Para el ciclo escolaren curso al momento de la observación, el taller de LSM se estaba desarrollando pero con los alumnos, con los compañeros de grupo de Itzel, lo cual, desde el punto de vista de la maestra Maty ha sido muy bueno, porque ha propiciado que ellos se interesen por interactuar y comunicarse con su compañera. Como punto de contraste refiere cómo eran las interacciones con el grupo de compañeros de Itzel del ciclo escolar anterior, con los cuales cursó prácticamente toda la primaria: "Porque, comentaba con la Maestra de Lenguaje que el año pasado, a pesar de que eran niños que habían convivido mucho más tiempo con ella, no veíamos tanta interacción". De esta manera resalta lo que ha significado que el grupo actual si tenga ese acercamiento a la LSM:

Entonces nosotros vemos esa diferencia de los años anteriores a ahora, que los niños como que intentan siempre estarse comunicando con ella y sobre todo más por aprender LSM; preguntan o tratan de hacer alguna seña para que ella también entienda y también tenga la confianza de tratar de comunicarse con ellos. Entonces ahorita, por ejemplo, veo que va y toca a cualquier niño, o se ríe con ellos, o algo están haciendo, porque también luego empiezan a hacer travesuras, pero el año pasado era menos. (MAESTRA DE GRUPO, 21/02/18)

El impacto positivo del taller de LSM, tanto para Itzel como para sus compañeros, es también reconocido por la Directora:

Y ahorita Itzel ya empieza a ser más interactiva, ya empieza a comunicarse y como tienen un taller de lenguaje de señas los niños, la Maestra de Lenguaje y la Maestra de Grupo, pues ya hay una mayor interacción. Ahorita como ya algunos niños si saben algunas señas, pues sí. Por ejemplo, a veces preguntan: -y ¿cómo se dice que les presten sus tijeras? o ¿qué si tiene esto? - Y ya otro le dice: -dile gracias-. Y pues ya saben cómo decir gracias. (23/02/18)

Durante las observaciones de clase se registraron variados momentos en los que Itzel interactuaba con algunos compañeros en LSM, a veces por iniciativa de ella y a veces de ellos. Incluso, se les observó haciéndose de repente algunas travesuras, eran momentos que ella parecía disfrutar de manera especial. En una ocasión uno de los alumnos me enseñó cómo saludar a Itzel y me comentó que cuando no saben cómo expresar algo en LSM, le piden a ella que les enseñe. Para eso, por ejemplo, le muestran un dibujo de un objeto sobre el cual quieren saber cómo se "dice".

Es importante mencionar que, según la maestra Maty, de alguna manera también se ha impactado a los otros niños de la escuela que no toman el taller, pues ellos, "ya saben que al menos existe otro lenguaje para ella". Eso de alguna manera favorece el reconocimiento y aceptación de su compañera: "todos los compañeros tratan de comunicarse con ella, aunque no saben cómo".

La importancia de las interacciones de Itzel con diversos integrantes de la comunidad escolar es 
fundamental, antes que nada para su desarrollo emocional y social, pero también para su aprendizaje. Dos grandes contribuciones de Vigotsky (1979), permiten afirmar esto. En primer lugar, su planteamiento sobre la importancia de las relaciones sociales en la conformación de la actividad, tanto "interna" como "externa" de los individuos. En este sentido, se desprende que las interacciones entre los alumnos en el espacio escolar facilitan y potencializan el desarrollo y el aprendizaje, no sólo de Itzel, sino de quienes se involucren en ellas. En segundo lugar, su tesis sobre el carácter mediado de la acción humana y la definición del signo como instrumento para influir en la conducta tanto del otro como de sí mismo, permite mirar las interacciones con Itzel como acciones mediadas por un sistema de signos (LSM), que permite la comunicación y establecimiento de relaciones sociales. Esta se veía limitada por la no disponibilidad de un sistema compartido de comunicación. Los signos brindan la posibilidad de influirse, de modificarse mutuamente. En este sentido, los beneficios son para todos los implicados.

\section{Las múltiples mediaciones}

Otro aspecto importante de trabajo de USAER con Itzel es el apoyo de la Maestra de Lenguaje en el aula, quien además actúa como intérprete de LSM. La maestra Lili, en adelante así la llamaré, entra al aula los lunes, martes y miércoles, aunque a veces falta debido a que tiene que desarrollar otras actividades con su equipo de la USAER, ya también atienden a otros alumnos y brindan orientación a maestros y padres de familia. El apoyo de la maestra Lili en el grupo de Itzel inició el ciclo escolar anterior, pero, según la maestra Maty, es en éste cuando más avances ha notado en su alumna.

El trabajo que realiza la maestra Lili con Itzel a veces se articula a la clase que la maestra Maty desarrolla con todo el grupo, aunque con algunas adaptaciones considerando las necesidades especificas de la alumna. Pero, otras veces, se desarrolla con actividades extras, al margen de lo que hace el grupo, dando prioridad al aprendizaje de la lectura, la escritura y contenidos básicos de matemáticas: "ya dijimos que le vamos a dar más prioridad a los conceptos básicos de matemáticas, sobre todo la lectura de números, las operaciones básicas, porque ya se va a ir a la secundaria" (MAESTRA DE GRUPO, 21/02/18).

Para la maestra Maty, el trabajo con la lectura, escritura y lo básico de matemáticas es la prioridad, pues son las herramientas básicas para que Itzel pueda aprender otras asignaturas: "Si, en eso nos vamos a centrar más. Ya lo demás yo pienso que ella lo va a aprender, es que ella es muy inteligente, aprende bien rápido. Si nosotros le diéramos las herramientas necesarias ella aprendería mucho". Este énfasis es sin duda comprensible y encuentra sustento en la importancia que Vigotsky atribuyó a las herramientas del pensamiento en la vida consciente del ser humano. Sin embargo, es importante señalar la manera cómo en la escuela estaban trabajan ese proceso:

Estamos en ese proceso (de que Itzel aprenda a Leer), creo que sí hemos avanzado, porque por ejemplo, nosotros hemos visto que ella, por lo mismo de que no escucha ningún sonido, pues no podría aprender como los otros niños normales. Entonces nosotros creemos que lo que ella ha aprendido y va a aprender es memorístico, es una situación muy memorística. Ellos tienen que retener mucho o memorizar muchas palabras para que así pueda asociar con otras palabras. Por ejemplo, digamos los inicios, a veces le digo: que escriba pera, entonces le tengo que decir que es como la papa y ya le hago la seña, entonces ella tienen que estar asociando las palabras. (MAESTRA DE GRUPO, 21/02/18)

Vigotsky llegó a afirmar que el potencial de desarrollo de los niños con deficiencia debería enfocarse en las funciones psicológicas superiores y que como estos se desarrollan en la acción social compartida con otros mediante el uso de herramientas culturales, entonces debemos concentrar nuestros esfuerzos en ajustar esos medios a sus diferentes necesidades y no restringirse a enseñar habilidades simples con base en recursos visuales, sino también con base en un pensamiento abstracto (VAN DER VEER; VALSINER, 2009).

Además de trabajo en grupo, Itzel recibe una atención individualizada de parte de la Maestra de Apoyo y del Psicólogo, ambos del equipo de USAER, aunque a veces esto implica sacar a la niña del grupo: "En el caso de Itzel, la mayor parte de la atención es que la maestra de lenguaje este en el grupo, pero también tiene un trabajo individualizado y ahí es en donde se ubican en las aulas de USAER". (DIRECTORA, 23/02/18)

El equipo de la Supervisión de Educación Especial de la zona escolar, también desarrolla una acción de apoyo a la educación de Itzel, orientada a favorecer sus procesos de desarrollo y socialización más allá de la escuela: 
Ellos, como equipo de USAER de la zona, tienen un proyecto, en donde cada mes tienen programadas reuniones con todos los niños que tienen problemas de audición. Ellos van creando unos contextos, por ejemplo, hace unos meses fue aquí en la comunidad, tocó el contexto del restaurant. Entonces vino aquí un muchacho que imparte LSM, con todos los maestros y los niños, nos enseñó el vocabulario para estar en un restaurant y luego fuimos al de aquí de la comunidad. Vinieron todos los niños y cada quien pidió su comida y entonces eso le ha ayudado a Itzel. Ahorita creo que ayer tuvieron el del centro comercial, entonces ella fue ahora al centro comercial, igual para hacer la interacción y así están buscando otros contextos. (DIRECTORA, 23/02/18).

En conversación con el Supervisor y una Asesora Técnico Pedagógica, ambos de Educación Especial, comentaron el sentido de este proyecto. Se trata de abrir espacios para que los alumnos con discapacidad auditiva vayan aprendiendo a desenvolverse en diferentes contextos de la vida social y comunitaria. Por otro lado, se trata de ir consolidando una pequeña comunidad de personas sordas. Este tipo de acciones son un buen ejemplo de cómo desde un espacio escolar se trata de crear oportunidades de "enculturación" de los niños, particularmente de quienes presentan alguna discapacidad.

De forma adicional e independientemente de la escuela, la niña asiste una vez por semana a la institución filantrópica donde le enseñaron LSM. Se trata de una asociación civil, que trabaja con niños con discapacidad para, entre otras cosas, brindarles capacitación laboral: "les ayudan sobre todo a aprender alguno oficio, algo que a ellos, en un momento dado, pueda servirle para generar sus propios recursos y poder sostenerse". (DIRECTORA, 23/0218) Aunque esto se hace en el horario escolar, por lo que la niña falta una vez a la semana.

La importancia de que los contextos sociales e institucionales generen ambientes y condiciones que favorezcan la socialización y enculturación de la alumna con sordera es sin duda fundamental. Vigotsky planteó que los alumnos con alguna deficiencia requieren de una educación basada en la organización particular de sus funciones y en sus características más positivas, en vez de sus aspectos deficitarios. Esto sólo puede lograrse en el marco de un sistema social y educativo que propositivamente atienda las necesidades específicas de estos alumnos. En este sentido, el caso analizado es ejemplo de una experiencia local donde diferentes actores se comprometen y tratan de construir posibilidades para el desarrollo de Itzel. La dificultad está en cómo articular estos esfuerzos en un proyecto educativo común, que no interfiera en una escolarización inclusiva.

\section{Contrastando condiciones áulicas: las clases de ciencias naturales}

Desde mi primera incursión en el aula, identifico inmediatamente a Itzel, principalmente porque su ubicación se destaca con respecto a sus compañeros de grupo. Ellos están sentados en bancas individuales pegadas a tres de las paredes del salón, formando una especie de herradura. Itzel se encuentra al final de uno de los brazos de la herradura, junto al escritorio de la maestra de grupo. Pero, a diferencia de sus compañeros, ella tiene una mesa y una silla y se observan diferentes materiales en el lugar. Además, en algunas clases, junto a ella se encuentra sentada una joven con la que interactúa en LSM, se trata de Lili, su Maestra de Lenguaje.

De las ocho clases observadas, Itzel asistió a cuatro (debido a que asiste a capacitación laboral) y de esas cuatro, en dos contó con el apoyo de la maestra Lili, pero en las otras dos fue la maestra de grupo, Maty, quien asumió sola la responsabilidad de trabajar con Itzel al mismo tiempo que con el resto del grupo. Esto brinda la posibilidad de contrastar lo que sucede con esta alumna en dos condiciones diferentes (con el apoyo y sin el apoyo de la Maestra de Lenguaje).

En las dos clases que no contaron con el apoyo de la Maestra de Lenguaje, la maestra Maty asigna a Itzel actividades diferentes a las de sus compañeros. Mientras la maestra desarrolla la clase de ciencias con el resto del grupo, Itzel se mantiene al margen, trabajando en su cuaderno, ya sea en tareas de escritura o de cálculo matemático. De cuando en cuando la maestra monitorea el trabajo de su alumna, dándole algún tipo de retroalimentación o nuevas indicaciones a través de algunas señas. En momentos de trabajo más autónomo por parte del grupo, la maestra se sienta junto a Itzel a trabajar con ella en el cuaderno.

Como ya se dijo, las prioridades de trabajo con Itzel son la lectura, la escritura y contenidos básicos de matemáticas. Pero además, la maestra Maty externa en diferentes ocasiones la dificultad de trabajar con esta alumna, debido a que sus otros estudiantes le demandan mucha atención. En el grupo anterior de Itzel había 23 niños, lo cual 
dificultó mucho atenderla, así que para este ciclo escolar decidieron tener un grupo más reducido:

Se opto por hacer el grupo de 11 niños, incluida ella, ¿para qué? Pues ella necesitaba más atención, que sí se puede trabajar con ella, pero teniendo menos niños y que también los niños que tuvieran fueran un poquito más independientes, que no requirieran mucho tiempo de mi. (MAESTRA DE GRUPO, 21/02/18).

Al parecer, para la maestra Maty, el trabajo que debe hacer con Itzel es individualizado, pero no lo puede hacer del todo por tener a otros alumnos que también requieren mucho de su atención, aún cuando sean poquitos:

Pero pues la verdad no resultó, porque los alumnos que llegaron, ósea el grupo que yo tenía de 11, con la mayoría yo tenía que estar. Entonces no funcionó, no hubo mucha ayuda para Itzel, porque eran poquitos, pero de todos modos me quitaban mucho tiempo, mucha atención, entonces ella es la que requiere más. (MAESTRA DE GRUPO, 21/02/18).

Recientemente (hacía unas dos semanas), el grupo aumentó a 18 , debido a que otra maestra de sexto no pudo seguir frente a su grupo y entonces repartieron a los alumnos entre los dos grupos de sexto restantes, uno de ellos el de la maestra Maty. Esto dificultó más ese trabajo más individualizado que la maestra supone debe hacerse.

En estas clases, observé a Itzel a veces trabajando en lo suyo, pero por momentos con cierto aburrimiento y mirando de cuando en cuando lo que hacían sus compañeros. Un dato interesante fueron dos momentos en los que la maestra mostró al grupo y manipuló un espejo de auto (un retrovisor), ejemplificando algo sobre el tema de la luz. En esos momentos, la mirada y el interés de Itzel se dirigían hacia la clase, curiosa por lo que hacían con ese objeto. Sin embargo, esta situación, de manipular algún objeto, fue aislada, ya que en estas clases predominó más la interacción oral y el trabajo con el libro de texto y el cuaderno.

En las dos clases donde sí estuvo la maestra Lili, la dinámica fue diferente, no sólo por la presencia de ésta, sino por la inclusión de actividades experimentales, con el manejo de variados objetos. En la primera de estas clases, la maestra Maty inicia el trabajo con su grupo: presenta el tema, lo escribe en el pizarrón (La importancia de las transformaciones temporales y permanentes de los materiales), explica de qué se trata, subrayando algunas palabras; lo vincula con temas vistos previamente, pone algunos ejemplos de la transformación de materiales y va planteándoles preguntas al respecto. Después, les pregunta si trajeron los materiales que les pidió para trabajar: hielo, mantequilla, barro. Les comenta que van a observar lo que pasa con estos, pero que antes copien las preguntas que vienen en el libro (relacionadas con las manipulaciones experimentales que van a realizar).

Mientras la clase se desarrolla de esa manera, Itzel y la maestra Lili parecen mantenerse al margen, interactuando en LSM entre ellas y trabajando otro tipo de actividades en el cuaderno. Esto es así hasta el momento en que sus compañeros, conformados en equipos, salen del salón a realizar las actividades experimentales, sobre amplias mesas de madera bajo la sombra de algunos árboles. Pasa un poco de tiempo antes de que Itzel y la maestra Lili salgan a observar lo que hace uno de los equipos. Esta alumna observa por un rato cómo una de sus compañeras amasa barro, después se regresa al salón, mientras la maestra Lili va detrás de ella para continuar con lo que estaban trabajando.

Después de algunos minutos, ellas vuelvan a salir, curiosas por lo que hacen los equipos y en esta ocasión entablan interacción con uno de ellos. Una de las compañeras les explica lo que hacen, mientras que la maestra Lili lo traduce a LSM. Itzel se ve interesada y se va animando a tocar el barro, primero lo hace de forma tímida, pero poco a poco se atreve a más: lo toca con un dedo y se limpia en la ropa, de repente ya mete la mano, luego ya toma una cierta cantidad, juega con él, embarra a la maestra, lo comienza a amasar, ríe, se ve contenta. Adicionalmente, observa lo que otros hacen y en algún momento, explora también uno de los libros de ciencias que están sobre la mesa donde vienen las indicaciones sobre las actividades.

Bajo la supervisión de la Maestra de Grupo, el equipo realiza otra manipulación experimental: queman una hoja de papel, mientras ella les pregunta sobre lo que pasa. Itzel observa atenta y la maestra Lili le explica lo que ocurre. La maestra Maty indica al equipo que contesten las preguntas que copiaron en su cuaderno con base en lo que observaron con las actividades. Itzel observa un poco y luego se mete al salón con la mestra Lili.

En la otra clase con la presencia de la Maestra de Lenguaje, la curiosidad e interés de Itzel por el trabajo de ciencias naturales se vuelve a manifestar, sobre todo porque el mayor tiempo de la clase se dedica a elaborar y probar un dispositivo experimental. 
Mientras la maestra Maty comienza a desarrollar la clase con el resto del grupo, la maestra Lili trabaja con Itzel una actividad diferente, al margen de la dinámica grupal. En diferentes momentos, esta alumna muestra interés en lo que sucede con sus compañeros, mirando por ejemplo, cómo la maestra Maty los está organizando en equipos para desarrollar una actividad experimental. La maestra Lili, responde a esta curiosidad, explicándole en LSM lo que está ocurriendo. Por su parte, la maestra Maty, al advertir el interés de su alumna, le indica a la maestra Lili que la integre a uno de los equipos. Sin embargo, Itzel se resiste a cumplir la indicación, hasta que llega un momento en que la maestra Lili jala la banca donde la alumna está sentada para acercarla un poco al equipo, la resistencia continúa, hasta que uno y después otro de sus compañeros la van jalando en varios momentos, así, poco a poco la integran físicamente al equipo. Itzel sólo se deja llevar, al parecer divertida y contenta con la situación.

La maestra Lili, revisa el libro, observa y escucha las indicaciones de la maestra Maty, tratando de comprender lo que se va a hacer para a su vez explicárselo a su alumna en LSM: se trata de construir un dispositivo conocido como "cámara obscura", un artefacto que permite reflejar dentro de la caja la imagen de un objeto del exterior de forma invertida ${ }^{6}$. Itzel observa lo que hacen sus compañeros de equipo y manipula los materiales. Llega un momento en que la maestra Lili se enfoca en ayudar a la maestra Maty a probar uno de los artefactos ya construidos, entonces Itzel, al igual que otros niños, se acerca a observar atentamente los variados intentos de las maestras por hacer que el dispositivo funcionara. Mientras algún compañero observa por la cámara oscura, ella también se asoma, tratando de observar algo. En otro momento, ya sin las maestras, es ella quien sostiene el objeto que se pretende observar y más tarde, sostiene la lámpara dirigiendo la luz hacia el objeto. Con expresiones gestuales le indica a uno de sus compañeros que observe por el orificio de la caja. En otro momento toma uno de los libros de ellos y observa la página donde viene la actividad. Se acerca al dispositivo, parece que le dice algo a su compañero en LSM sobre el funcionamiento de la caja. Luego regresa otra vez a observar el libro. La clase se interrumpe por ser la hora del recreo.

Al terminar el recreo, continua la misma dinámica, con Itzel igual de curiosa por lo que pasa, tratando de participar en las pruebas con el artefacto, interactuando con sus compañeros e incluso jugando en algunos momentos con ellos. Esto continua así hasta que el Psicólogo de USAER se la lleva para tener su sesión individual de trabajo en otro espacio.

Las descripciones presentadas pueden analizarse desde muchos ángulos, pero aquí quiero destacar cómo cada situación plantea condiciones diferenciales para la participación y aprendizaje de Itzel. En las clases donde sólo está la maestra Maty, la interacción de esta alumna se limita a breves momentos de trabajo con ella, alrededor de una actividad desarrollada de manera individual, al parecer tediosa o aburrida para ella. Sin embargo, Itzel muestra algunos momentos de curiosidad e interés por lo que sucede en la clase, sobre todo cuando se manipula algún tipo de material.

En las dos clases donde la maestra de lenguaje está presente, las posibilidades de interacción se amplían, pues la mediación realizada a través de la LSM, permite que la actividad individual esté apoyada y acompañada por ésta. En la primera clase descrita, la curiosidad e interés de Itzel por el contexto y dinámica social del aula la lleva a implicarse un poco más es la actividad grupal, con la mediación de la intérprete. Sin embargo, se mantiene como observadora, con algunos intentos de asumir un papel más activo, manipulando alguno de los materiales, mientras que la maestra Lili, trata de traducirle el significado de lo que ocurre con las actividades experimentales.

En la segunda clase, con la presencia de la Maestra de Lenguaje, nuevamente la curiosidad e interés de Itzel propician que la maestra Maty abandone en ese momento la idea de mantenerla en un trabajo individual, al margen del grupo y centrado en las asignaturas "prioritarias". Al dejar un poco a Itzel con cierta autonomía (pues las maestras estaban concentradas en hacer funcionar el dispositivo), la alumna se fue implicando cada vez en la actividad grupal, tomando diferentes iniciativas para apoyar la realización de las manipulaciones experimentales, interactuando de forma más intensa con sus compañeros y tratando de entender lo que se estaba trabajando (inferido por su curiosa mirada sobre el libro impreso).

Es importante notar, que Itzel y sus compañeros entablaron interacciones diversas, no sólo para "socializar", sino para implicarse en la actividad académica. Los instrumentos mediadores no se limitaron a lo que los niños han aprendido de la LSM, usaron también otros recursos multimodales para comunicarse, tales como los gestos indicativos, las miradas y hasta las sonrisas.

No es posible saber qué es lo que Itzel 
aprendió en cada situación, pero si atendemos la tesis de Vigotsky respecto a que son las condiciones de la vida social las que posibilitan el desarrollo de la actividad del individuo, tanto interna como externa, podemos inferir que entre mayor sea la riqueza de las relaciones sociales, mayores serán las posibilidades de su aprendizaje y desarrollo.

\section{Consideraciones finales}

El análisis realizado permitió identificar en la trayectoria escolar de Itzel condiciones institucionales que se fueron transformando a partir de la llegada de una nueva Directora escolar. La visión educativa, social y política de esta figura la llevó a movilizar los escasos recursos institucionales disponibles en su región. La modalidad de apoyo de educación especial para la inclusión de alumnos con discapacidad en escuelas regulares, como otros recursos y servicios educativos, no tienen la cobertura necesaria, sobre todo en zonas rurales y en las comunidades más alejadas de las ciudades. Por eso no es extraño que los primeros años de escolarización de Itzel hayan transcurrido sin el apoyo de la USAER.

A pesar de que la política de inclusión de alumnos con discapacidad en escuelas regulares se formalizó hace más de veinte años, es frecuente encontrar escuelas que, como la de Itzel, con muy buena disposición y voluntad, reciben a estos niños sin estar preparadas, sin tener una capacitación específica para atenderlos ni con los recursos de apoyo que se recomiendan en los documentos internacionales que promueven la educación inclusiva (UNESCO, 1990, 1994; ONU, 2006). Y aún así, en el caso de análisis, se identificaron esfuerzos significativos por ofrecer algo a su alumna con sordera, aunque no supieran muy bien cómo hacerlo, tal como lo reconoció su maestra.

Es importante destacar la importancia de la familia en el proceso de desarrollo y aprendizaje de la alumna. A temprana edad, su madre promovió su atención en una asociación filantrópica, donde le hicieron disponible un sistema de signos, una herramienta tan fundamental para la comunicación, el pensamiento, la vida social y consciente, tal como lo señaló Vigotsky. El que la alumna llegara a la escuela primaria con el dominio de la LSM sin duda fue una condición favorable, que, sin embargo, no podía ser aprovechada dado que en este lugar no había "hablantes" de la misma lengua, produciendo un relativo aislamiento de la niña. En este sentido, puede decirse que, aun estando en la escuela de su comunidad, con sus congéneres, tal como se promueve desde la educación inclusiva, la alumna permanecía excluida de muchos aspectos de la vida social y académica de ese escenario.

La transformación de las condiciones de la escuela incluyó en primer lugar la promoción de actitudes favorables para la inclusión de todo el alumnado en las actividades académicas y sociales, con énfasis especial en el caso de Itzel. En segundo lugar, pero de manera fundamental, el apoyo de la USAER, en particular de la Maestra de Lenguaje, al promover que diferentes miembros de la comunidad escolar se fueran apropiando de la lengua que Itzel domina. Con esto abrió posibilidades para enriquecer las relaciones sociales de esta alumna en la escuela y el aula. Su actuación como intérprete también se convirtió en una condición importante para avanzar en la enseñanza de los contenidos prioritarios, definidos por los profesionales implicados (Directora, maestras, USAER). De manera colateral, se observaron momentos en donde la curiosidad de la alumna presionaron para llegar a participar en la clase de ciencias naturales.

Particularmente quiero destacar cómo las actividades experimentales, propias del trabajo con las ciencias naturales, ofrecen un escenario social y académico atractivo para Itzel (y en general para todos los alumnos). La riqueza de recursos semióticos (como las representaciones visuales en el libro), de objetos, de artefactos, de actividades, de fenómenos implicados en la enseñanza de la ciencia, presenta posibilidades de participación, interacción y desarrollo de habilidades tales como la propia curiosidad, la observación, comparación, relaciones causa-efecto, etc. Esto es, representa una oportunidad excelente para promover procesos de aprendizaje y desarrollo. Desafortunadamente, la participación de la alumna en esta y otras asignaturas queda relegada por el interés en atender las prioridades definidas, no sólo por los actores escolares, sino por el Sistema Educativo Mexicano.

No niego la trascendencia del aprendizaje de la lectura, la escritura y contenidos básicos de matemáticas, desde el legado de Vigotsky se fundamenta claramente su importancia. Sin embargo, desde una aproximación sociocultural, la apropiación de los sistemas de signos, de las herramientas simbólicas de la cultura, se produce en la relación con los objetos, las personas, los artefactos, la operación con los signos y en el marco de las actividades sociales. En este sentido, entre más rico y organizado sea el escenario social y cultural para la instrucción, así como las actividades que se implican, las 
posibilidades de aprendizaje y desarrollo de los niños serán mayores.

Para Vigotsky, la educación formal era "el instrumento esencial de enculturación y humanización" (RIVIÉRE, 1985, p. 18). La escuela es el espacio sociocultural con mayores posibilidades de influencia en el aprendizaje y desarrollo de los individuos. Para los alumnos con discapacidad, a veces representa la única opción para el desarrollo de su potencial humano. Su escolarización en las instituciones educativas comunes, es sin duda un gran avance. Los esfuerzos de las escuelas, de los docentes y de las familias por ofrecer algo a estos niños son sumamente valiosos $y$ deben ser reconocidos. Sin embargo, los sistemas educativos aún tienen una gran deuda en la generación de las condiciones necesarias para que en estas escuelas sea viable una educación inclusiva.

\section{Notas}

1 Un agradecimiento especial a la comunidad escolar, su Directora, Maestra Karime Ramírez López, y a la Maestra de Grupo por permitirme indagar su valioso trabajo.

2 Los nombres, tanto de la alumna como de sus maestras, fueron cambiados para preservar su anonimato.

3 Las USAER (Unidades de Servicios de Apoyo a la Escuela Regular), constituyen un servicio de apoyo a la escolarización, entre otros, de alumnos con discapacidad en las escuelas comunes.

4 En las escuelas de educación básica en México, antes de iniciar el ciclo escolar cada colectivo docente elabora un plan que proyecta las estrategias y acciones que implementarán para mejorar el servicio educativo que brindan.

5 Como parte del equipo de la USAER, la Maestra de Lenguaje trabaja para favorecer la comunicación. En el caso que se presenta, la maestra tiene formación como intérprete en LSM.

6 Es una actividad incluida en el Libro de Texto Gratuito de Ciencias Naturales de Sexto Grado, como parte del tema, "Aprovechamiento de la formación de imágenes en espejos y lentes" (SEP, 2014, p. 117).

\section{Referencias}

COLE, M.; SCRIBNER, S. Introducción. En: VYGOTSKY, L. El desarrollo de los procesos psicológicos superiores. Barcelona: Grijalbo, 1979, pp. 17-36.

JUÁREZ, J. M.; COMBONI, S.; GARNIQUE, F. De la educación especial a la educación inclusiva. Argumentos, UAM X, Núm. 62, p. 41-83, 2010

LURIA, A. R. Conciencia y lenguaje. Madrid: Aprendizaje Visor, 1984.

MÉXICO. Secretaría de Educación Pública. Ley General de Educación. Diario Oficial de la Federación, 13/07/1993. Disponible en: http://www.diputados.gob.mx/LeyesBiblio/ref/lge/L GE_orig_13jul93_ima.pdfAcceso el 29 junio 2018.

. Proyecto General para la Educación Especial en México. Cuadernos de Integración Educativa.n. 1. México: Dirección de Educación Especial, SEP, 1994.

Decreto por el que se reforman y adicionan diversas disposiciones de la Ley General de Educación, en materia de Educación Inclusiva. Diario Oficial de la Federación, 01/06/2016. Disponible en:

<http://www.dof.gob.mx/nota_detalle.php?codigo= $5439577 \&$ fecha $=01 / 06 / 2016>$ Acceso en 29 junio 2018.

MEZA, G.C.L. La educación de los niños y las niñas con discapacidad en México: algunos elementos para su análisis. En: DEL RIO, L. N. $L a$ primera infancia en el espacio público. Experiencias latinoamericanas. México: UAM, 2015, p. 69-85

NARANJO, F. G. B. El trabajo docente en el marco de las políticas de educación inclusiva.

Aproximación a partir de un estudio de caso. $C P U-e$ Revista de Investigación Educativa, Núm. 24,

Xalapa, p. 99-124, 2017

ORGANIZACIÓN DE LAS NACIONES UNIDAS (ONU). Convención de los derechos de las personas con discapacidad. Nueva York: 2006.

ORGANIZACIÓN DE LAS NACIONES UNIDAS PARA LA EDUCACIÓN, LA CIENCIA Y LA CULTURA (UNESCO). Declaración Mundial de Educación para Todos. Jomtién:1990. . Declaración de Salamanca y Marco de 
Acción para las Necesidades Educativas Especiales. Salamanca: 1994.

RIVIÉRE, A. La psicología de Vygotsky. Madrid: Aprendizaje Visor, 1985

ROCKWELL, E. Etnografía y teoría en la investigación educativa, Enfoques. En: Congreso del Tercer Seminario Nacional de Investigación en Educación. Centro de Investigaciones de la Universidad Pedagógica, Bogotá, 1986.

ROCKWELL, E. La experiencia etnográfica: historia y cultura en los procesos educativos. Buenos Aires: Paidós, 2009.

\section{SECRETARÍA DE EDUCACIÓN PÚBLICA} (SEP). Ciencias Naturales. Sexto grado. $3^{\mathrm{a}}$. ed. México: 2014.

SOARES, F. M. R.; LACERDA, C. B. F. O aluno Surdo em escola regular: um estudo de caso sobre a construção da identidade. En: GÓES, M. C. R.; LAPLANE, A. L. F. (Orgs.). Políticas e Práticas de educação Inclusiva. 3. ed. Campinas: Autores
Associados, 2007,p. 121-147.

TERIGI, F. Trayectorias escolares e inclusión educativa: del enfoque individual al desafío para las políticas educativas. En: MARCHESI, A.; BLANCO R.; HERNÁNDEZ, L. (Coords.). Avances y desafíos de la educación inclusiva en Iberoamérica. Madrid: Organización de Estados Iberoamericanos para la Educación, la Ciencia y la Cultura, 2014, p. 71-87.

VAN DER VEER, R.; VALSINER, J. Vygotsky: uma Síntese. 6a. Ed. São Paulo: Edições Loyola, 2009.

VYGOTSKY, L. El desarrollo de los procesos psicológicos superiores. Barcelona: Grijalbo, 1979. WERTSCH, J. Vygotsky y la formación social de la mente. Barcelona, Buenos Aires, México: Paidós, 1988.

WERTSCH, J.; TULVISTE; VALSINER. A sociocultural approach to agency. En: FORMAN, E.; NORRIS, M.; STONE, A. (Eds.) Contexts for learning. New York: Oxford University Press, 1993, p. 336-356.

\section{Sobre a autora}

Gabriela Begonia Naranjo Flores: Doctora en Ciencias con Especialidad en Investigaciones Educativas y Profesora de Tiempo Completo en la Universidad Pedagógica Nacional, México.

Recebido em julho de 2018.

Aprovado em outubro de 2018. 